\title{
The Effects of Repetitive Transcranial Magnetic Stimulation Integrated Mirror Therapy on the Gait of Chronic Stroke Patients
}

\author{
Hyun-Gyu Cha ${ }^{1}$ and Myoung-Kwon Kim ${ }^{2 *}$ \\ ${ }^{1}$ Department of Physical Therapy, Kyungbuk College, Yeongju 750-050, Korea \\ ${ }^{2}$ Department of Physical Therapy, College of Rehabilitation Sciences, Daegu University, Gyeongsan-si 712-714, Korea
}

(Received 1 April 2015, Received in final form 14 April 2015, Accepted 15 April 2015)

\begin{abstract}
This study was conducted to determine the effects of repetitive transcranial magnetic stimulation (rTMS) integrated mirror therapy on the gait of post-stroke patients. Thirty patients who were six months post-stroke were assigned to either the experimental group $(n=15)$ or the control group $(n=15)$. Stroke patients in the experimental group underwent rTMS and mirror therapy for the lower limbs, while those in the control group underwent rTMS and sham therapy. Participants in both groups received therapy five days per week for four weeks. A significant difference in post-training gains for the single support phase, step length, stride length and velocity was observed between the experimental group and the control group $(p<0.05)$. The experimental group showed a significant increment in the single support phase, step length, stride length, swing phase, velocity, cadence, double support phase and step width as compared to pre-intervention $(p<0.05)$. The control group showed a significant increment in step length, velocity, cadence and step width compared to preintervention $(p<0.05)$. Further investigation of the availability and feasibility of rTMS integrated mirror therapy for post-stroke patients as a therapeutic approach for gait rehabilitation is warranted.
\end{abstract}

Keywords : transcranial magnetic stimulation, gait, mirror therapy

\section{Introduction}

Stability control of the body trunk and strong driving force of the lower limbs are required during walking for patients who have suffered strokes. However, due to the damage to their central nervous systems, their walking action shows defects in balancing and posture control. As such, compensation of the lower limb of the non-paralyzed side occurs, in order to compensate for the motor control ability of the lower limb of the paralyzed side. This causes difficulty in maintaining a normal walking action [1]. Approximately two-thirds of stroke survivors have a walking disability [2]. The main differences in their walking, compared to that of ordinary people, includes a decrease in walking velocity and steps per minute, a reduction of step length, and imbalance between the lower limbs [3]. Abnormal walking resulting from this affects their ability to perform independent daily activity, and disease prognosis among patients with stroke [4].

CThe Korean Magnetics Society. All rights reserved.

*Corresponding author: Tel: +82-53-850-4352

Fax: +82-53-850-4353, e-mail: kimmk@daegu.ac.kr
Hence, an independent walking ability for patients who have suffered strokes is considered one of the most important factors in their functional recovery [5]. Intervention methods for the functional recovery of such patients have been largely limited to the indirect and functional induction of changes in the brain. Among diverse physiotherapeutic approaches that have recently been developed, therapy methods based on brain neuroplasticity regarding the recovery capability of the central nervous system have come to the fore, following advancements in science.

Repetitive transcranial magnetic stimulation (rTMS) is a method of noninvasive stimulation of the damaged area of the brain, whereby stimulation of the brain can be performed either selectively or directly $[6,7]$.

Mirror therapy is one of the intervention methods for walking recovery. The lower limb of the non-paralyzed side of a patient who has suffered a stroke is moved in order to induce a visual illusion through the reflection in a mirror, such that the patient feels as if his or her paralyzed lower limb is moving in an ordinary manner. Mirror therapy is based on the principle of neural plasticity [8].

Studies on rTMS and mirror therapy have mainly focused 
on the functional recovery of the upper limbs among stroke patients. In particular, those in the sub-acute stage where neurological recovery occurs rapidly used to constitute the majority of the sample.

Moreover, studies combining visual illusion effects with direct stimulation of the brain that has effects on the functional recovery of stroke patients are rare. Hence, this study attempts to investigate how mirror therapy accompanied by rTMS affects the walking ability of chronic stroke patients.

\section{Materials and Methods}

Thirty subjects participated in the experiment. The Research Ethics Committee of Eulji University Hospital approved the study, and all participants provided informed, written consent prior to their involvement. When the initial assessment was completed, the subjects were randomly assigned either to an experimental group ( $n=$ $15)$ or to a control group $(n=15)$. Subjects in the experimental group received rTMS and mirror therapy for a total of 40 minutes per day. Subjects in the experimental group received this training five days per week for four weeks. Subjects in the control group received rTMS and sham therapy for a total of 40 minutes per day on the same day.

Magstim Rapid2 (Magstim Co Ltd, Wales, United Kingdom) was used for rTMS, and a figure-of-eight coil with a diameter of $70 \mathrm{~mm}$ was used. For measurement of motor-evoked potential, recording electrodes were attached on the tibia anterior muscle of the paralyzed side lower limb, which is laterally $2 \mathrm{~cm}$ from the anterior margin of the tibia. The reference electrode was attached $30 \mathrm{~mm}$ from the recording electrode and parallel with the direction of muscle fiber. The ground electrode was attached to the center of the top of the foot of the same side.

After finding a spot with the strongest motor-evoked potential at the lowest level, the spot was stimulated 10 times. Peak amplitude over $50 \mu \mathrm{V}$ more than five times was defined as the resting motor threshold. Stimulation was at $90 \%$ of the resting motor threshold and, based on the EG 10/20 system, the central lobe area of the cerebral hemisphere was stimulated at a frequency of $10 \mathrm{~Hz}$ for ten seconds followed by resting for 50 seconds. This process was repeated 20 times, where a total of 20 minutes were spent [8]. This device is reported to have high testretest reliability $(\mathrm{r}=0.60)$ and validity $(\mathrm{r}=0.92)$ [9].

Mirror therapy was performed using a modified version of that described in the study of Sütbeyaz et al. [9]. A mirror was mounted on a stand, tilted toward the paretic side of the body to prevent the participant from viewing the paretic limb.

For the experimental group, the reflective surface was kept facing the non-paretic leg. The exercises were performed in a semi-seated position and were: (1) hip-knee-ankle flexion; (2) knee extension with ankle dorsiflexion, and; (3) knee flexion beyond 90 degrees. The control group performed the same exercises and for the same duration as the experimental group, but the reflective side of the mirror was covered with white fabric. Both groups performed only non-paretic lower limb movements during mirror therapy. Participants did not move their paretic limb.

Temporospatial gait data were obtained using a sixcamera motion analysis system (Eagle system, Motion Analysis, Santa Rosa, CA, USA) sampling $120 \mathrm{~Hz}$. Hemispherical or flat reflective markers $(25 \mathrm{~mm}$ diameter) were applied at the following sites on both sides of the body: anterior superior iliac spine, superior aspect of the L5sacral interface, thigh (lower thigh below the midpoint), medial femoral condyle, lateral femoral condyle, shank (lower shank below the midpoint), medial malleolus, lateral malleolus, posterior calcaneus, center of the foot between the second and third metatarsals. To facilitate application and visualization of the markers, each of the subjects wore a pair of skin-tight cycling shorts and a sleeveless top.

EvaRt and Orthotrak software (Motion Analysis, Santa Rosa, CA, USA) were used to process the data. After a test walk to become accustomed to the experimental procedure, the subjects were asked to walk along a $7 \mathrm{~m}$ walkway for three sessions, and the average values of the data were used. The temporospatial gait characteristics were evaluated, including step length, stride length, rate of swing phase, rate of stance phase, velocity, cadence and step length, using a motion analysis device [3].

Differences in the general characteristics between the experimental group and the control group before therapy were compared using independent $t$-tests and chi-square tests. A paired samples $t$-test was used for comparisons of gait before and after training within each group. An independent $t$-test was used for comparisons of pre-test and post-test differences in gait between the experimental group and the control group. Statistical analysis was performed using statistical software SPSS 18.0 (SPSS, Chicago, IL, USA). The level of significance was chosen as 0.05 .

\section{Results}

A summary of the clinical and demographic features of the sample $(n=30)$ is shown in Table 1, which also shows 
Table 1. General and medical characteristics of subjects.

\begin{tabular}{lcc}
\hline \hline & EG $(n=15)$ & CG $(n=15)$ \\
\hline Age (years) & $59.43 \pm 13.00^{\mathrm{a}}$ & $62 \pm 12.00$ \\
Sex (male/female) & $7 / 8$ & $6 / 9$ \\
Duration (month) & $14.45 \pm 3.14$ & $14.13 \pm 1.55$ \\
Weight (kg) & $67.67 \pm 8.06$ & $64.73 \pm 5.66$ \\
Height (cm) & $166.27 \pm 7.03$ & $162.87 \pm 6.56$ \\
Causes (infarction/hemorrhage) & $11 / 4$ & $9 / 6$ \\
Affected side (right/left) & $8 / 7$ & $10 / 5$ \\
MMSE-K (score) & $27.26 \pm 1.78$ & $26.61 \pm 2.26$ \\
\hline
\end{tabular}

${ }^{a}$ Mean \pm SD, EG: rTMS + Mirror Therapy, CG: rTMS + sham Therapy, MMSE-K, Mini-Mental State Examination

that there were no significant differences in the baseline characteristics between the two groups $(p>0.05)$. A significant difference in post-training gains for the single support phase, step length, stride length and velocity was observed between the experimental group and the control group $(p<0.05)$. The experimental group showed a significant increment in the single support phase, step length, stride length, swing phase, velocity, cadence, double support phase and step width as compared to pre-intervention $(p<0.05)$. The control group showed a significant increment in the step length, velocity, cadence and step width as compared to pre-intervention $(p<0.05)$.

\section{Discussion}

This study was conducted to investigate the effect of mirror therapy with rTMS on improvement in the gait ability of patients with stroke. After four weeks of mirror therapy with rTMS, significant improvements in gait ability were observed between the experimental group and the control group. This result supports the primary hypothesis of the study: that mirror therapy with rTMS would improve the gait ability of stroke patients. rTMS is one of the new approaches of clinical intervention for recovery of exercise function among patients with stroke, which noninvasively stimulates the cerebral cortex to cause depolarization in brain cells. This implies that the iterative stimulation of rTMS changes irritability of the cerebral cortex, which contributes to the functional recovery of patients with stroke [7,9].

In a mirror therapy, the cerebral cortex on the paralyzed side of patients with stroke is activated as the relevant brain cells of the patient respond to actions of other people as if the patient is carrying out the action by himself or herself by just looking at it. This is called a mirror neuron, which plays a critical role in understanding the behavior of other people or learning about
Table 2. Comparison of change in temporospatial gait characteristics of the experimental group and control group.

\begin{tabular}{cccc}
\hline \hline & EG $(n=15)$ & CG $(n=15)$ & $t$ \\
\hline Single support phase (\%) & & & \\
Pre-test & $25.38 \pm 9.68$ & $24.66 \pm 8.69$ & 0.22 \\
Post-test & $35.54 \pm 9.44$ & $27.03 \pm 7.88$ & $2.68^{* *}$ \\
$t$ & $-4.18^{* *}$ & -1.58 & \\
Change value & $10.16 \pm 9.39$ & $2.37 \pm 5.78$ & $2.73^{* *}$ \\
Double support phase (\%) & & & \\
Pre-test & $71.35 \pm 5.83$ & $70.61 \pm 6.39$ & 0.33 \\
Post-test & $65.01 \pm 6.46$ & $67.15 \pm 6.41$ & -0.91 \\
$t$ & $4.92^{* *}$ & 1.96 & \\
Change value & $-6.34 \pm 4.98$ & $-3.46 \pm 6.80$ & -1.32 \\
Step length (cm) & & & \\
Pre-test & $29.52 \pm 9.96$ & $28.52 \pm 7.34$ & 0.31 \\
Post-test & $39.75 \pm 6.78$ & $32.72 \pm 6.67$ & $2.86^{* *}$ \\
$t$ & $-3.77^{* *}$ & $-3.79^{* *}$ & \\
Change value & $10.23 \pm 10.50$ & $4.20 \pm 4.28$ & $2.06^{*}$ \\
Stride length (cm) & & & \\
Pre-test & $58.31 \pm 16.36$ & $60.52 \pm 23.57$ & -0.30 \\
Post-test & $81.84 \pm 10.31$ & $68.52 \pm 21.53$ & $2.16^{*}$ \\
$t$ & $-6.45^{* *}$ & -1.57 & \\
Change value & $23.53 \pm 14.12$ & $8.00 \pm 19.71$ & $2.48^{*}$ \\
\hline
\end{tabular}

${ }^{\mathrm{a}}$ Mean \pm SD, EG: rTMS + Mirror Therapy, CG: rTMS + sham Therapy

${ }^{\mathrm{b}}$ Effect size greater than $0.70,{ }^{*} p<.05,{ }^{* *} p<.01$,

Table 3. Comparison of change in temporospatial gait characteristics of the experimental group and control group (Continue).

\begin{tabular}{cccc}
\hline \hline & EG $(n=15)$ & CG $(n=15)$ & $t$ \\
\hline Swing phase (\%) & & & \\
Pre-test & $29.33 \pm 6.23$ & $30.73 \pm 5.47$ & -0.65 \\
Post-test & $33.79 \pm 3.74$ & $32.45 \pm 6.48$ & 0.69 \\
$t$ & $-2.95^{* *}$ & -0.96 & \\
Change value & $4.46 \pm 5.84$ & $1.72 \pm 6.91$ & 1.17 \\
Velocity (cm/s) & & & \\
Pre-test & $36.39 \pm 16.82$ & $36.38 \pm 18.67$ & 0.10 \\
Post-test & $53.67 \pm 13.81$ & $41.92 \pm 16.62$ & $2.10^{*}$ \\
$t$ & $-4.13^{* *}$ & $-2.66^{* *}$ & \\
Change value & $17.27 \pm 16.19$ & $5.54 \pm 8.06$ & $2.51^{*}$ \\
Cadence (step/min) & & & \\
Pre-test & $51.91 \pm 11.52$ & $54.45 \pm 13.74$ & -0.55 \\
Post-test & $61.14 \pm 11.74$ & $58.33 \pm 15.01$ & 0.57 \\
$t$ & $-2.75^{* *}$ & $-2.13^{*}$ & \\
Change value & $9.22 \pm 12.96$ & $3.87 \pm 7.05$ & 1.40 \\
Step width (cm) & & & \\
Pre-test & $17.38 \pm 2.45$ & $18.56 \pm 3.62$ & -1.04 \\
Post-test & $10.02 \pm 3.33$ & $11.75 \pm 3.85$ & -1.32 \\
$t$ & $8.49^{* *}$ & $5.44^{* *}$ & \\
Change value & $-7.37 \pm 3.36$ & $-6.81 \pm 4.84$ & -0.37 \\
\hline
\end{tabular}

${ }^{\mathrm{a}}$ Mean \pm SD, EG: rTMS + Mirror Therapy, CG: rTMS + sham Therapy

${ }^{\mathrm{b}}$ Effect size greater than $0.70,{ }^{*} p<.05,{ }^{* *} p<.01$ 
new techniques [10].

In this study, a spatio-temporal walking analysis was performed for measurement of the independent walking ability of patients with stroke. According to the main results of this study, the experimental group showed more effective recovery of walking ability than the control group in all of the walking variables, including single support phase, step length, stride length, and velocity. It is conjectured that rTMS accompanied by mirror therapy activated the ascending and descending corticospinal tract of the spinal cord and increased proprioceptive sense, balancing sense, and irritability of the neuron network of the relevant motor cortex, which eventually contributed to the enhancement of walking ability [11].

The double support phase is the stage where the center of gravity of the body is most stable. Patients with problems in balancing tend to have an increased double support phase due to the slowdown in walking speed [12]. Hence, it is conjectured that the cerebrum and cerebellum, which control balance, were activated in the experimental group, which subsequently stimulated the proprioceptive sense and related neuron network. This eventually increased the walking speed, causing a decrease in double support phase.

Allen et al. [13] reported that when step length increases during walking, swing phase increases along with stride length. Neumann [12] reported that the step length of normal adults is approximately $71 \mathrm{~cm}$ and stride length is approximately $142 \mathrm{~cm}$. In this study, the experimental group showed increased step length and stride length, but it showed no difference from the normal length. This can be interpreted as patients with stroke having decreased stride length and step length due to defects in balancing ability, and length of lower limbs varies among individuals. In addition, it is surmised that the increase in velocity and cadence are caused by the increased swing phase and step length from the patients' recovery of balance.

Due to unstable center of weight, the length of step width among patients with stroke is usually longer than that of ordinary people. This implies that acquisition of a balanced base of support is important in walking recovery among patients with stroke. As mirror therapy accompanied by rTMS increases the stance phase time of the lower limb on the paralyzed side while increasing the swing phase of the lower limb of the non-paralyzed side, it is expected to aid the recovery of balanced walking. Moreover, as a result of increased stability of the lower limb of the paralyzed side, the ability to control the passenger portion consisting of head, body trunk and upper limbs has also been reinforced.

rTMS is reported to influence long-term potentiation and long-term depression relevant to the brain neural plasticity [14]. In case of application of rTMS, long-term potentiation that increases the connection of synapses is particularly related to motor learning and memory ability. Centripetalism input on the motor cortex has been reported to increase the potential capacity of synapse [14].

Sütbeyaz et al. [8] used a sample of 40 patients, less than 12 months after each had suffered a stroke, and randomly allocated them into a mirror therapy group and a control group. The lower limb of the non-paralyzed side was placed in front of a mirror on which ankle dorsiflexion was iteratively performed for 30 minutes a day, five days a week, for a total of four weeks. According to the results, the mirror therapy group showed significant improvement in exercise function and walking ability (functional ambulation categories; FAC), compared to the control group. The results are similar to those of this study. It is conjectured that the mobility of the paralyzed side was improved as the visual illusion of the mobility of the non-paralyzed side through the mirror reflection was transferred to the brain of the stroke patients. In addition, visual information through a mirror also helps patients with stroke to better recognize body displacement and orientation in a space [15].

Hence, for functional recovery of patients with stroke, mirror therapy accompanied by selective and direct rTMS stimulation on the damaged cerebral cortex can be an effective intervention method for the recovery of their walking ability.

The current study has some limitations. First, the small sample size may have influenced certain variables and influenced the results. Therefore, these results cannot be generalized to all stroke patients. Second, the absence of follow-up after completion of repetitive transcranial magnetic stimulation did not allow for determining the durability of the effects of this intervention. Third, functional measures were not undertaken to determine whether or not there would be a functional benefit in terms of activities. Hence, further studies, including a long-term follow-up assessment, are needed to evaluate the longterm benefits of repetitive transcranial magnetic stimulation in conjunction with mirror therapy.

\section{References}

[1] L. Bensoussan, S. Mesure, J. M. Viton, and A. Delarque, J. Rehabil Med. 38, 287 (2006).

[2] M. Shauqhnessy, K. M. Michael, J. D. Sorkin, and R. F. Macko, Stroke. 36, 1305 (2005).

[3] M. Roerdink, C. J. Lamoth, G. Kwakkel, P. C. van Wierinqen, and P. J. Beek, Phys. Ther. 87, 1009 (2007). 
[4] H. Barbeau and M. Visintin, Arch. Phys. Med. Rehabil. 84, 1458 (2003).

[5] P. Duncan, S. Studenski, L. Richards, S. Gollub, S. M. Lai, D. Reker, S. Perera, J. Yates, V. Koch, S. Riqler, and D. Johnson, Stroke. 34, 2173 (2003).

[6] K. Sugawara and T. Kasai, Hum. Mov. Sci. 21, 203 (2002).

[7] N. Sasaki, S. Mizutani, W. Kakuda, and M. Abo, J Stroke Cerebrovasc Dis. 22, 413 (2013).

[8] S. Sütbeyaz, G. Yavuzer, N. Sezer, and F. Koseoglu, Arch. Phys. Med. Rehabil. 88, 555 (2007).

[9] S. C. Livingston and C. D. Ingersoll, Int. J. Neurosci. 118, 239 (2008).
[10] G. Rizzolatti and L. Craighero, Annu. Rev. Neurosci. 27, 169 (2004).

[11] V. Di Lazzaro, M. Dileone, P. Profice, F. Pilato, B. Cioni, M. Meqlio, F. Capone, P. A. Tonali, and J. C. Rothwell, Stroke. 37, 2850 (2006).

[12] D. A. Neumann, Mosby, Philadelpia (2002).

[13] J. L. Allen, S. A. Kautz, and R. R. Neptune, Gait Posture. 33, 538 (2011).

[14] A. Berardelli, M. Inghilleri, J. C. Rothwell, S. Romeo, A. Curra, F. Gilio, N. Moduqno, and M. Manfredi, Exp. Brain Res. 122, 79 (1998).

[15] C. Dohle, J. Pullen, A. Nakaten, J. Kust, C. Rietz, and H. Karbe, Neurorehabil Neural Repair. 23, 209 (2009). 